\title{
TB blurs the lines
}

Mycobacterium tuberculosis has long been thought to reside within an intact membrane-bound phagosome in host cells, but recent papers add weight to the suggestion that this is not the only mode of survival for this important intracellular pathogen.

In 2007, Peters and colleagues used cryo-immunogold electron microscopy of the human THP-1 macrophage cell line infected with the virulent strain $M$. tuberculosis str. H37Rv to show that the bacterium escaped into the cytosol and that this escape was dependent on the ESX-1 secretion system. Writing in reconstituted cytosolic translocation and enhanced virulence.

This work examined the intracellular fate of $M$. tuberculosis after infection for at least 2 days. However, writing in Cell Host \& Microbe, Cox and colleagues provide evidence that permeabilization of the phagosome membrane occurs at a much earlier stage. These authors analysed the transcriptional response of mouse bone marrow-derived macrophages to infection with $M$. tuberculosis str. Erdman, and identified an ESX-1-dependent regulon that included many interferon regulatory signalling pathways showed that the STING-TBK1-IRF3 pathway was activated and, moreover, that the ligand responsible was extracellular DNA. The authors hypothesize that extracellular DNA from lysed bacteria can leak from the phagosome into the cytosol when the phagosomal membrane has been perforated. Evidence of ESX-1-dependent phagosomal rupture was also found in a PLoS Pathogens paper that was published earlier in 2012, in which Brosch, Enninga and colleagues used a FRETbased assay to look at the intracellular localization of M. tuberculosis.
Cellular Microbiology, they now follow up on this paper by examining the intracellular localization of a wide range of non-pathogenic and pathogenic mycobacterial species. They found that all pathogenic species examined, including clinical isolates, encoded a functional ESX-1 system that secreted ESAT6 $(6 \mathrm{kDa}$ early secretory antigenic target), which has been shown to perturb membranes, and they all escaped from the phagosome. ESX-1 is encoded in a genomic region known as region of difference 1 (RD1), and the authors demonstrated that restoring RD1 in an avirulent mycobacterial strain factor 3 (IRF3)-dependent interferonstimulated genes. This transcriptional response was reminiscent of the response to infection with the cytosolic pathogen Listeria monocytogenes, so the authors used fluorescence resonance energy transfer (FRET) to assess the intracellular localization of M. tuberculosis in their system. They found that the phagosome membrane was permeabilized as early as 3 hours after infection and that permeabilization, and hence activation of cytosolic innate immunity, was dependent on ESX-1. A subsequent detailed characterization of the downstream immune
These results show that there can be continuity between the phagosome and the cytosol, thus blurring the lines that distinguish vacuolar from cytosolic pathogens. Clearly, we still have much to learn about this complex host-pathogen relationship.

Sheilagh Molloy

ORIGINAL RESEARCH PAPERS Houben, D. et al. ESX-1-mediated translocation to the cytosol controls virulence of mycobacteria. Cell. Microbiol. 17 Apr 2012 (doi:10.1011/j.1462-5822.2012.01799.x)| Manzanillo, P. S. et al. Mycobacterium tuberculosis activates the DNA-dependent cytosolic surveillance pathway within macrophages. Cell Host Microbe 11, 469-480 (2012)|Simeone, R. et al. Phagosomal rupture by Mycobacterium tuberculosis results in toxicity and host cell death. PLoS Pathog. 8, e1002507 (2012) 